\title{
Nutritional Imbalances in Wildlife
}

\author{
Nichole Rosenhagen, DVM \\ PAWS WILDLIFE CENTER \\ SEATTLE, WASHINGTON
}

\begin{abstract}
Nutritional disorders are uncommon in free-living wild animals. However, animals in captivity are at much higher risk for developing nutrition-related disease, often as a result of an unnatural habitat and diet.

Angel wing, thiamine deficiency, perosis, metabolic bone diseases, capture myopathy and hypovitaminosis A are some of the most common nutritional imbalances wildlife rehabilitators are likely to encounter. Successful management of these conditions requires a thorough understanding of the pathophysiology of each disease as well as the clinical signs, treatment options, and most importantly, prevention strategies.
\end{abstract}

Keywords: Nutrition, imbalance, angel wing, thiamine, perosis, metabolic bone disease, capture myopathy, vitamin A

\section{INTRODUCTION}

In free-living wild animals, nutritional imbalances are uncommon. In their natural environment, animals generally consume balanced diets and have access to sunlight. However, animals that end up in captivity or those that live in highly urbanized environments are much more likely to develop disease related to nutrition. Urban sprawl increases the likelihood of human-wildlife interactions and often gives wild animals easy access to artificial and imbalanced diets in the form of garbage and well-meaning handouts. Thus, as humans continue to encroach into the natural habitat of wild animals, nutritional imbalances will undoubtedly become more prevalent. The following review will provide information about some of the most common pathologic conditions related to nutrition in wild animals.

\section{ANGEL WING}

Angel wing, also known as slipped wing, airplane wing, or dropped wing, is a deformity of the growing metacarpal bones that leads to rotation of the primary flight feathers so that they protrude when the wing is folded (Flinchum 2006). Angel wing is likely a multifactorial condition, but is most often a result of

Nichole Rosenhagen graduated from the University of Illinois College of Veterinary Medicine in 2015. After graduation, she worked for two years in the University's Wildlife Medical Clinic. Nicki is now a full time veterinarian at the PAWS Wildlife Center in Lynnwood, WA. a diet containing excessive protein. Genetics, calcium/ phosphorus, and/or vitamin D imbalance, manganese deficiency, vitamin E deficiency, and inadequate exercise may all play a role in the development of the condition as well (Kear 1986). Regardless of the specific etiology, the result is a disproportionate growth rate between the feathers and the musculoskeletal structures of the wing. The relatively heavy weight of the growing blood feathers places excessive force on the underdeveloped muscles and ligaments of the carpus which causes the wing to twist outward (Zsivanovits et al 2006). Affected birds are often juvenile waterfowl that should naturally consume diets with a crude protein of not more than 17 to 18 percent, especially cygnets and goslings, though the condition has been documented in other species, including raptors and psittacines (Zsivanovits et al 2006). The condition may develop when these species are fed diets formulated for smaller, faster growing species like mallard ducklings. Thus, rehabilitators who house ducklings and goslings together should be vigilant about monitoring the patient growth rates and wing carriage. Any signs of wing drooping or twisting of the primary flight feathers can be early indication of a problem and may require a change in husbandry.

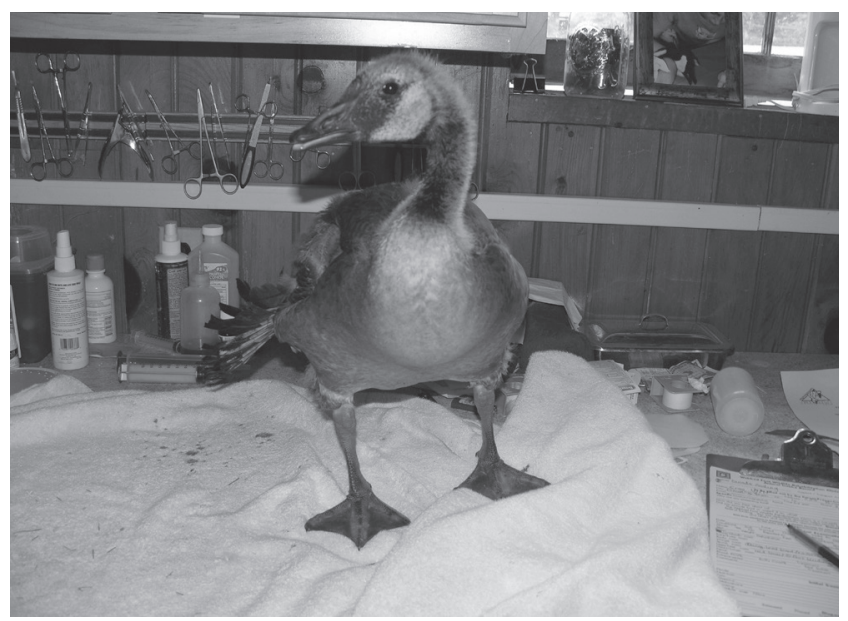

Figure 1. Juvenile Canada goose (Branta canadensis) with angel wing. Photo by Michele Goodman. 


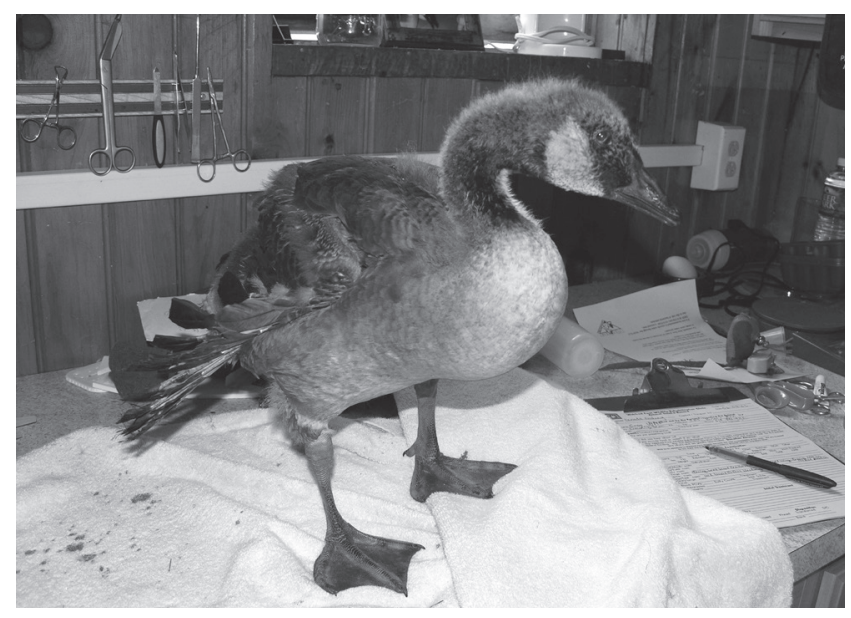

Figure 2. Juvenile Canada goose (Branta canadensis) with angel wing. Photo by Michele Goodman.

Angel wing is diagnosed based on diet history and clinical signs. If a diagnosis is made early, conservative treatment may be corrective. Bandaging or taping the wing in a normal position for several days followed by range of motion therapy may be effective. The caretaker should take note to only keep the wing bandaged for three to five days at a time to prevent muscle contracture and propatagial ligament damage (Flinchum 2006). Care should also be taken to not bend, break, or otherwise damage the growing blood feathers with wrapping materials. Additionally, the underlying etiology should be corrected if possible. For chronic conditions, surgical repair is necessary, though the prognosis for flight is poor and euthanasia should be considered.

Shell pyramiding in chelonians is the nutritional equivalent of angel wing. Pyramiding is the

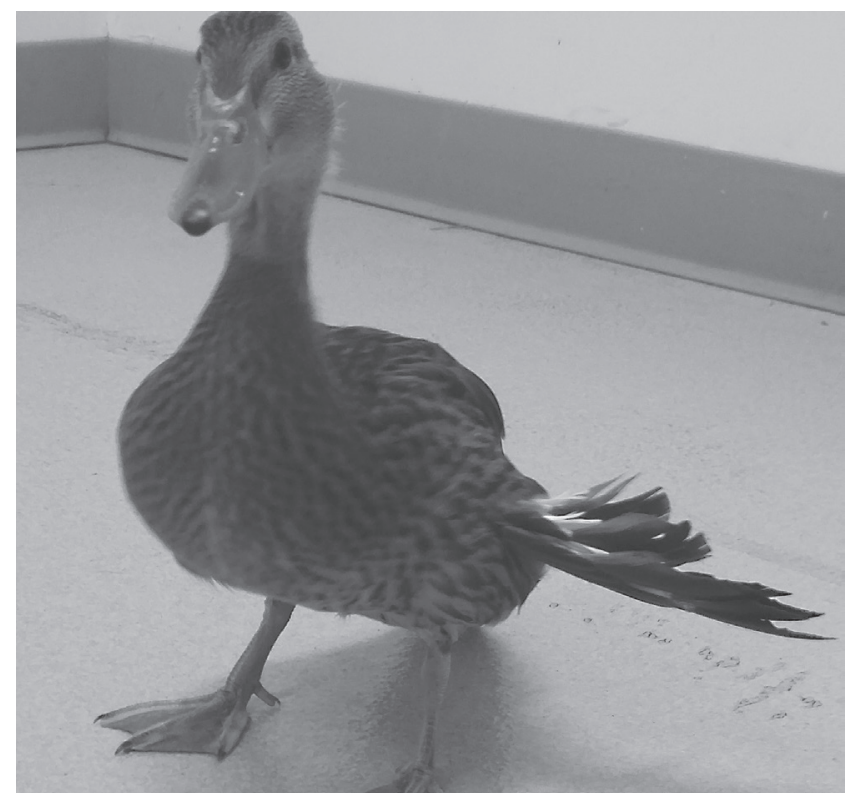

Figure 4. Angel wing in a six week old duckling. Photo by Renée Schott.

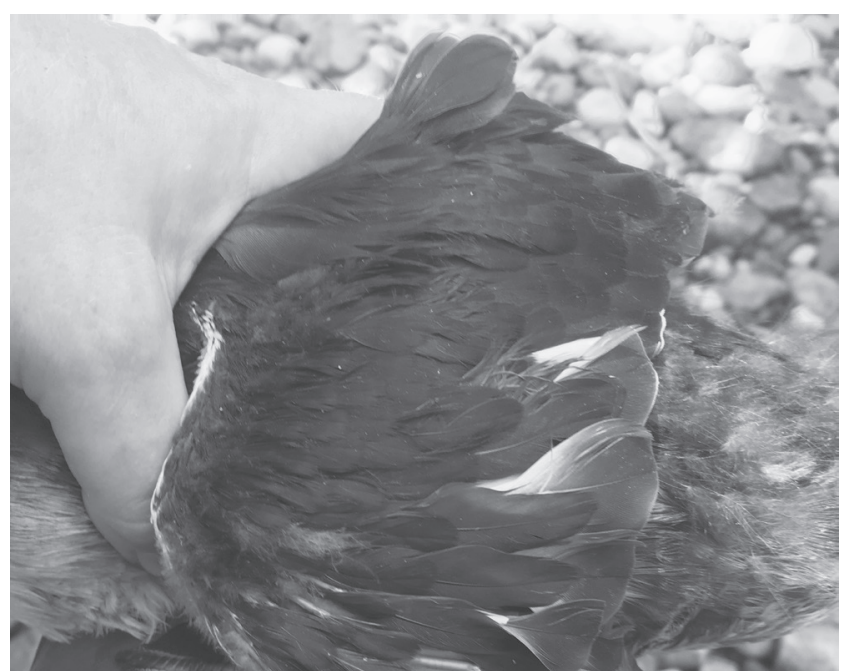

Figure 3. Droop wing in a six week old duckling. Photo by Michele Goodman.

development of symmetrical, pyramid-shaped bony growths of the carapace. Like angel wing, the suspected cause is multifactorial, but likely involves excessive caloric intake and a subsequent increase in growth rate. Herbivorous tortoises are particularly susceptible to this condition (Chitty and Raftery 2013). Management is based on exercise and slowing the growth rate via diet and environmental changes (Donoghue 2006).

\section{THIAMINE DEFICIENCY}

Thiamine (vitamin B1) is a water-soluble vitamin that plays many important roles in the body. It is essential for carbohydrate, amino acid, and fatty acid synthesis and metabolism, and it acts as a cofactor for various enzymes that produce antioxidants, nucleic acids, hormones, neurotransmitters, and adenosine triphosphate (ATP). The brain is particularly reliant on thiamine for energy metabolism and neurotransmitter synthesis (Wedekind et al 1994; Robbins 1983). Thiamine is absorbed from food in the small intestine, and requirements increase when metabolism increases. Hence, conditions that decrease absorption or increase the body's metabolism, such as intestinal disease, pregnancy, and infection, put an animal at risk for deficiency. Thiamine deficiency can also occur as a result of decreased intake. Processed foods with high quantities of simple carbohydrates have low levels of thiamine, as do day-old chicks (Carnarius et al 2008). Additionally, raw fish and shellfish may contain thiaminase, an enzyme that degrades the animal's store of thiamine within ninety minutes of its death (Croft et al 2013; Wedekind et al 1994). Hence, animals fed exclusively dead fish or chicks without thiamine supplementation are most likely to be affected by this condition. 
Due to its vast effects on the body, clinical signs of thiamine deficiency are non-specific. Animals may present with anorexia, weight loss, weakness, stargazing, tremors, ataxia, opisthotonus, seizures, and/or death (Fascetti and Delaney 2012; Doneley 2016; Wedekind et al 1994). A diagnosis is most often made based on diet history, clinical signs, and response to treatment, though histopathology of the brain may also be diagnostic (Croft et al 2013). The treatment of suspected thiamine deficiency involves administration of oral or injectable thiamine or vitamin B complex along with diet correction. In most cases, response to treatment is rapid, and as there is very little chance for toxicity, the reader is encouraged to supplement thiamine to all animals exhibiting clinical signs of non-specific neurologic disease as part of routine initial care.

\section{PEROSIS}

Perosis, also known as slipped tendon, is a luxation of the gastrocnemius (Achilles) tendon over the tibiotarsal condyles. This tendon displacement leads to apparent enlargement of the hock joint as well as twisting and/or shortening of the tarsometatarsus. Perosis is caused by a deficiency of dietary choline and/or manganese (Greenacre 2015). Birds require 10 to 12 times more dietary manganese than many mammals, so the condition most often manifests in juvenile birds, especially waterfowl (Wedekind et al 1994). Manganese and choline are important for normal

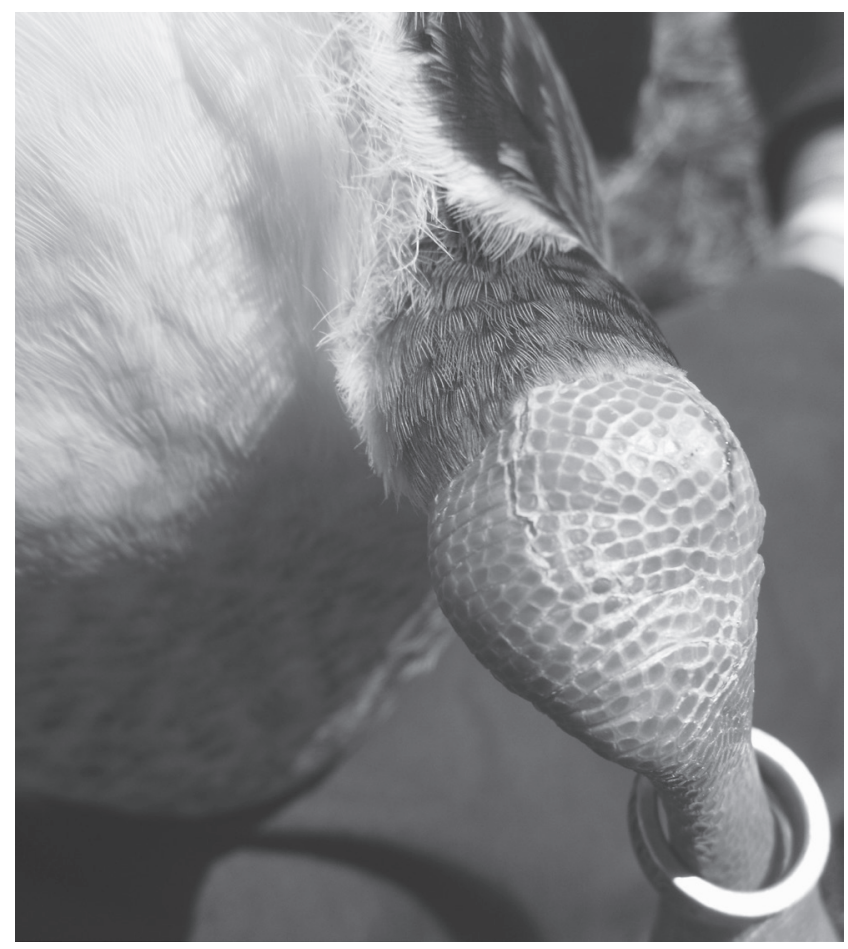

Figure 5. Medial deviation of the gastrocnemius tendon in a young goose. Photo by Michele Goodman. bone and cartilage formation, and animals that do not receive sufficient levels of these elements in their diets are predisposed to leg deformities which can allow the tendon to slip out of its natural groove in the hock. Once the tendon is deviated, it will contract, which accelerates the deformity and triggers inflammation (Graham 2016). Additional conditions that weaken the bones may also contribute to this disease.

Diet history and clinical presentation are used to make a diagnosis, and management varies with the severity of the condition. Early, mild cases may be managed with manual reduction, splinting, and diet correction (Goodman et al 2017). Severe or chronic cases often require surgical replacement and tacking of the tendon sheath to the bone (Greenacre 2015; Graham 2016). In either case, frequent rechecks and bandage changes are necessary to prevent additional problems as the bird grows. Unfortunately, the nature of the forces on the tendon often result in failure, so the prognosis for recovery is poor.

\section{Metabolic Bone Disease (NUTRITIONAL SECONDARY HYPERPARATHYROIDISM)}

Metabolic bone disease (MBD) is a term used to describe several disorders that affect the development, integrity, and function of bones. These conditions result from abnormal levels of calcium, phosphorous, vitamin $\mathrm{D}$, and/or parathyroid hormone. Most commonly, MBD is used in reference to nutritional secondary hyperparathyroidism (NSHP).

Nutritional secondary hyperparathyroidism, called rickets in young animals, occurs as a result of a deficiency of dietary calcium or vitamin $\mathrm{D}$, an excess of dietary phosphorous, and/or inadequate exposure to ultraviolet B (UVB) radiation (Mader 2006). Calcium is important for the structural integrity of bones and teeth, as well as for cell signaling, muscle contraction, blood clotting, and nerve transmission (Wedekind et al 1994). Vitamin D, which is synthesized by the body after exposure to the sun's UVB rays, facilitates intestinal absorption of calcium (Mader 2006). Phosphorous is also important for bone and tooth structure, but a balanced diet should contain an average ratio of two parts elemental calcium for each one part elemental phosphorus (by weight) for normal skeletal development (Wedekind et al 1994). Plant sources of phosphorus are often bound to other compounds such as phytate in a way that makes the phosphorus not nutritionally available, which may complicate calculation of dietary ratios. If the non-phytate phosphorus content of a plant source is not known, assume only $60-70 \%$ of phosphorus is available (Klasing 1999). 
In the event of an imbalance of one of these factors, calcium levels can become dangerously low. To prevent death secondary to acute hypocalcemia, the body has mechanisms to maintain physiologic levels in the blood. Specifically, low calcium levels trigger the release of parathyroid hormone to stimulate bone reabsorption (Mader 2006). While this is crucial for calcium homeostasis, the hormone's effect on the bones makes them weak and can lead to skeletal deformities and fractures. In severe or chronic cases when parathyroid hormone cannot correct the calcium imbalance, the animal may display anorexia, lethargy, weakness, dystocia or egg binding, constipation, seizures, or death (Mader 2006).

All animals are at risk of developing NSHP, though carnivorous animals appear to be less likely to develop the condition if they are given access to whole adult prey due to the presence of calcium in mature bones and cartilage. A diagnosis is most often made based on husbandry history and clinical signs. Radiographs may also reveal decreased bone density or abnormal bone conformation. Prior to detectable bone abnormalities, growing birds old enough to stand may appear painful when walking or show a reluctance to stand.

The reader should be aware that serum calcium levels are often within normal limits on a chemistry panel and should not be used to rule out the condition. Additionally, renal disease can lead to a similar condition, so kidney health should be evaluated in suspected cases of NSHP.

Treatment of NSHP involves supportive care, calcium supplementation under the direction of a veterinarian, and correction of any inappropriate husbandry. Even with treatment, bone deformities that have developed secondary to the disease will remain, so the animal's prognosis for long-term locomotion should be carefully considered prior to initiating treatment.

For prevention, animals in rehabilitation settings should receive a diet with the appropriate calcium to phosphorous ratio and have access to natural or synthetic UVB radiation. When UVB radiation is provided, there should be nothing between it and the animal. Plastic and glass both filter out the natural and synthetic rays and make them inaccessible. Additionally, feeder insects naturally contain low calcium and high phosphorous, so they should be dusted or gut loaded with a calcium supplement. Recommendations for correct insect supplementation are available (Livingston et al 2014).

\section{CAPTURE MYOPATHY}

Capture myopathy is a metabolic condition that can lead to significant morbidity and mortality, and all wild animals are susceptible. In most cases, capture myopathy results from prolonged pursuit or handling by humans. In these instances, extreme exertion by the animal being chased or restrained leads to an exhaustion of muscular ATP stores, decreased oxygen delivery to the tissues, and a buildup of lactic acid. This in turn leads to muscle necrosis and the release of free radicals and nephrotoxic myoglobin (Wolfe 2015). Capture myopathy also can be caused by nutritional deficiencies, especially vitamin $\mathrm{E}$ and selenium (Patterson 2007). Vitamin E and selenium help protect the body from reactive oxygen species and other free radicals that form during intense muscular exertion and trauma (Wedekind et al 1994). Deficient animals may be more likely to develop signs consistent with capture myopathy, including muscle stiffness and pain, ataxia, paresis, torticollis, paralysis, anorexia, or death (Patterson 2007). Deficiencies can occur secondary to a poor diet in captivity, but many areas of the country have soil that is naturally deficient in selenium; thus a wild animal's nutritional status may be compromised even before capture (USGS 2016).

In cases where capture myopathy is suspected, diagnosis is based on clinical signs, history of excessive exertion or poor diet, and serum chemistry changes consistent with muscle and kidney damage. On post mortem examination, muscle or renal necrosis can further support a diagnosis. Affected muscle may appear striped with light or whitish-colored bands. Unfortunately, treatment of capture myopathy after clinical signs manifest is often unsuccessful. When attempted, treatment should include vitamin E and selenium supplementation as well as analgesics, fluid therapy, low-stress handling, and when directed by a veterinarian, muscle relaxants such as diazepam or midazolam (Patterson 2007). Nutritional support may be needed as affected animals may have an impaired ability to eat. Vitamin $\mathrm{E}$ is fat-soluble, so toxicity is a concern during supplementation, particularly if an injectable formulation is used. Therefore, a veterinary formulary should be consulted for dosing information. As with any disease, prevention will be significantly more rewarding than management.

\section{HYPOVITAMINOSIS A}

Vitamin A is essential for many biologic functions. It is necessary for healthy epithelial tissue as well as normal vision, growth, reproduction, and immunity (Wedekind et al 1994). Animals suffering from hypovitaminosis A may display a variety of clinical signs 
including night blindness, anorexia, weakness, ataxia, and squamous metaplasia. Squamous metaplasia is often the most clinically apparent sign and refers to a change in the cell types of the epithelium. Cells that should be tall and mucus-producing become abnormally flattened, stacked, and keratinized, which can plug glands and lead to abscesses (Lawton 2006). Because epithelium can be found throughout the body, these changes have been documented in the respiratory, neurologic, gastrointestinal, and urogenital systems as well as the external skin. In birds, hypovitaminosis A frequently manifests as respiratory disease or hyperkeratosis of the beak and feet. Reptiles often present with swollen and infected eyes and ears. In amphibians, low levels of vitamin A are the suspected cause of a newly recognized condition called short tongue syndrome whereby the normally glandular, non-keratinized cells of the tongue undergo squamous metaplasia, limiting the animals' ability to apprehend and consume food (Pessier 2014). Mammals appear to be rarely affected by low vitamin A.

A diagnosis of hypovitaminosis A typically relies on clinical signs and diet history, and should be suspected in animals who receive all-seed or all-meat diets or those who consume unsupplemented insects. Additionally, research suggests that pesticides and other artificial chemicals in the environment may contribute to low vitamin $\mathrm{A}$ in free-living turtles (Holladay et al 2001). When a definitive diagnosis is required, vitamin A levels can be measured from liver biopsies, though established reference ranges are limited in many species, so interpretation of the results may be challenging.

Management of hypovitaminosis A relies on dietary correction and vitamin supplementation. As with vitamin $E$, vitamin $A$ is fat-soluble, and toxicities have been reported, so careful consideration regarding dose and route of supplementation is recommended.

\section{CONCLUSION}

Nutritional imbalances in free-living wild animals are rare, but increasing human-wildlife interactions will likely enhance their prevalence. Highlighted here are some of the more common presentations documented to date. This is by no means an exhaustive review as the full list of disorders related to nutrient deficits or excesses is considerably greater and likely not fully explored. When providing care to an animal with a suspected nutritional imbalance, the reader is encouraged to review the case thoroughly and attempt to rule out other, more likely causes of the animal's signs. When nutrition seems the most likely etiology, supportive care and diet correction will be the most beneficial treatment options. However, despite the apparently correctable nature of many of the described nutritional conditions, prevention will be a far more effective tool for the wildlife rehabilitator.

\section{LITERATURE CITED}

Carnarius, M., H. M. Hafez, A. Henning, H. J. Henning, and M. Lierz. 2008. Clinical Signs and Diagnosis of Thiamine Deficiency in Juvenile Goshawks (Accipiter gentilis). Veterinary Record. 163: 215-217.

Chitty, J., and A. Raftery. 2013. Shell Deformities. Pp. 309-312 in Essentials of Tortoise Medicine and Surgery. (J. Chitty and A. Raftery, editors). Wiley Blackwell: Chichester, West Sussex.

Croft, L., E. Napoli, C. K. Hung, J. St. Leger, S. Gearhart, K. Heym, S. Wong, D. Sakaguchi, A. Lin, B. Puschner, and C. Giulivi. 2013. Clinical Evaluation and Biochemical Analyses of Thiamine Deficiency in Pacific Harbor Seals (Phoca Vitulina) Maintained at a Zoological Facility. Journal of the American Veterinary Medical Association. 243(8): 1179-1189.

Doneley, B. 2016. Disorders of the Nervous System. Pp. 303-315 in Avian Medicine and Surgery in Practice: Companion and Aviary Birds. (B. Doneley, editor). Taylor and Francis Group: Boca Raton, FL.

Donoghue, S. 2006. Nutrition. Pp. 251-298 in Reptile Medicine and Surgery. (D. Mader, editor). Saunders Elsevier: St. Louis, MO.

Fascetti, A. J., and S. J. Delaney. 2012. Basic Nutrition Overview. Pp. 9-22 in Applied Veterinary Clinical Nutrition. (A. J. Fascetti and S. J. Delaney, editors). John Wiley and Sons: Chichester, West Sussex.

Flinchum, G. B. 2006. Management of Waterfowl. Pp. 831-847 in Clinical Avian Medicine, 2nd Volume. (G. J. Harrison and T. Lightfoot, editors). Spix Publishing: Palm Beach, FL.

Goodman, M., R. Schott, and R. S. Duerr. 2017. Waterfowl (Ducks, Geese, and Swans). Pp. 99-119 in Topics in Wildlife Medicine: Orthopedics. (R. S. Duerr and G. J. Purdin, editors). National Wildlife Rehabilitators Association: St. Cloud, $\mathrm{MN}$.

Graham, J. E. 2016. Splay Leg and Slipped Tendon. Pp. 272-273 in Blackwell's Five-Minute Veterinary Consult: Avian. (J. E. Graham, editor). John Wiley and Sons: Ames, IA.

Greenacre, C. B. 2015. Musculoskeletal Diseases. Pp. 145-159 in Backyard Poultry Medicine and Surgery: A Guide for Veterinary Practitioners. (C. B. Greenacre and T. Y. Morishita, editors). Wiley Blackwell: Ames, IA. (N.R日 
Holladay, S. D., J. C. Wolf, S. A. Smith, D. E. Jones, and J. L. Robertson. 2001. Aural Abscesses in Wild-Caught Box Turtles (Terapene Carolina): Possible Role of Organochlorine-Induced Hypovitaminosis A. Ecotoxicology and Environmental Safety. 48(1): 99-106.

Kear, J. 1986. Feeding and Nutrition. Pp. 335-341 in Zoo and Wild Animal Medicine, 2nd edition. (M. E. Fowler, editor). WB Saunders: Philadelphia, PA.

Klasing, K. C. 1999. Comparative Avian Nutrition. CAB International: Cambridge, MA.

Lawton, M. P. C. 2006. Reptilian Ophthalmology. Pp. 323-342 in Reptile Medicine and Surgery, 2nd edition. (D. R. Mader, editor). Saunders Elsevier: St. Louis, MO.

Livingston, S., S. R. Lavin, K. Sullivan, L. Attard, and E. V. Valdes. 2014. Challenges with Effective Nutrient Supplementation for Amphibians: A Review of Cricket Studies. Zoo Biology. 33(6): 565-576.

Mader, D. R. 2006. Metabolic Bone Diseases. Pp. 841-851 in Reptile Medicine and Surgery, 2nd edition. (D. R. Mader, editor). Saunders Elsevier: St. Louis, MO.

Patterson, J. 2007. Capture Myopathy. Pp. 115-121 in Zoo Animal and Wildlife Immobilization and Anesthesia. (G. West, D. J. Heard, and N. Caulkett, editors). Blackwell Publishing: Ames, IA.
Pessier, A. P. 2014. Short Tongue Syndrome and Hypovitaminosis A. Pp. 271-276 in Current Therapy in Reptile Medicine and Surgery. (D. R. Mader and S. J. Divers, editors). Elsevier Saunders: St. Louis, MO.

Robbins, C. T. 1983. Vitamins. Pp. 69-98 in Wildlife Feeding and Nutrition. Academic Press, Inc: Orlando, FL.

United States Geological Service (USGS). 2016. Selenium in Counties of the Conterminous States. Accessed Dec. 2016. Available from: <https://mrdata.usgs.gov/geochem/doc/averages/ se/usa.html>.

Wedekind, K. J., S. Yu, L. Kats, I. Paetau-Robinson, and C. S. Cowell. 1994. Micronutrients: Minerals and Vitamins. Pp. 107-148 in Small Animal Clinical Nutrition, 5th edition. (M. S. Hand, editor). Mark Morris Institute: Topeka, KS.

Wolfe, A. W. 2015. Bovidae (Except Sheep and Goats) and Antilocapridae. Volume 8: Pp. 626-645 in Zoo and Wild Animal Medicine. (R. E. Miller and M. E. Fowler, editors). Elsevier Saunders: St. Louis, MO.

Zsivanovits, P., D. J. Monks, and N. Forbes. 2006. Bilateral Valgus Deformity of the Distal Wings (Angel Wing) in a Northern Goshawk (Accipiter Gentilis). Journal of Avian Medicine and Surgery. 20(1): 21-26. (N. 장

\section{New NWRA Publication!!}

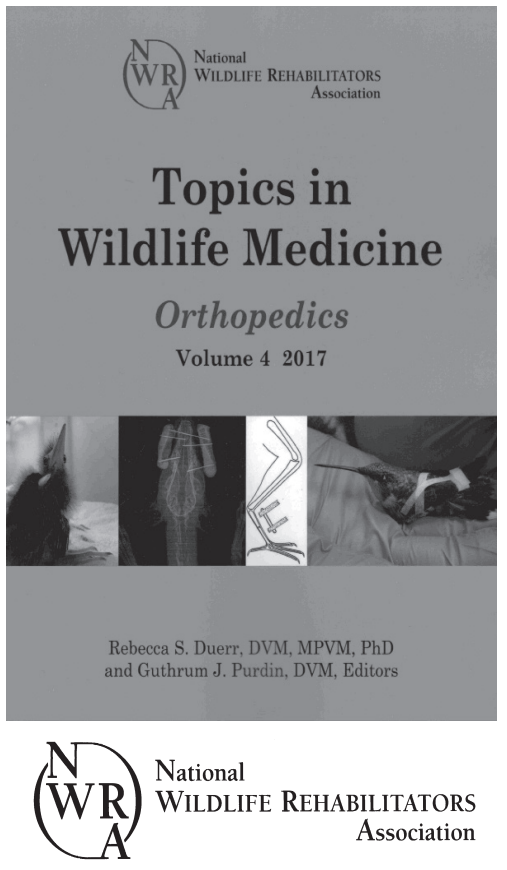

Topics in Wildlife Medicine Volume 4: Orthopedics was created to help veterinarians and wildlife rehabilitators manage fractures in native North American wildlife. From wraps and splints to surgical techniques and ethical considerations for different species, this book covers a lot of ground! The authors of each chapter are veterinarians and experienced wildlife rehabilitators who specialize in wildlife rehabilitation medicine. Both rehabilitators and their veterinarians will benefit from the tips, tricks, and techniques described within.

Topics in Wildlife Medicine Volume 4: Orthopedics is available to Members for $\$ 44.75$ (p\&h included), and Non-members for $\$ 50.75$ (p\&h included). For shipping to Canada or outside the US, please contact us for pricing.

To order your copy of Topics in Wildlife Medicine Volume 4: Orthopedics, please send a check or money order in US Dollars to:
NWRA Sales

2625 Clearwater Rd, Ste 110

St. Cloud, MN 56301
Call (320) 230-9920,

email us at NWRA@NWRAwildlife.org, or visit us online at www.NWRAwildlife.org. 